\title{
Macaulay's method for a Timoshenko beam
}

\author{
N. G. Stephen
}

School of Engineering Sciences, Mechanical Engineering, University of Southampton, Highfield, Southampton S017 1BJ, UK

E-mail: ngs@soton.ac.uk

Abstract The Macaulay bracket notation is familiar to many engineers for the deflection analysis of a Euler-Bernoulli beam subject to multiple or discontinuous loads. An expression for the internal bending moment, and hence curvature, is valid at all locations along the beam, and the deflection curve can be calculated by integrating twice with respect to the axial coordinate. The notation obviates the need for matching of multiple constants of integration for the various sections of the beam. Here, the method is extended to a Timoshenko beam, which includes the additional deflection due to shear. This requires an additional expression for the shearing force, also valid at all locations along the beam.

Keywords Macaulay; bracket notation; Timoshenko beam

\section{Notation}

A cross-sectional area

C constant of integration

E Young's modulus

$G \quad$ shear modulus

I second moment of area

$L \quad$ length of beam

M moment

$P \quad$ applied force

$Q \quad$ shearing force

$R \quad$ reaction force

$u, v$ displacement components in $x$ and $y$ directions

$w \quad$ uniformly distributed load

$x, y \quad$ planar Cartesian coordinates

$\kappa \quad$ shear coefficient

$\psi \quad$ cross-sectional rotation

\section{Introduction}

Macaulay's method is a familiar topic within many 'mechanics of solids' modules forming part of mechanical, aerospace and civil engineering undergraduate degree programmes [1]. The method is, in essence, a first exposure to (and pre-dates) generalised functions (e.g. Dirac delta, step, and ramp), with meaning given over to the bracket notation, typically of the form $[x-a]^{n}$; if the argument within the bracket is negative, that is, if $x<a$, the term is ignored, while if positive, that is, if $x>a$, it is treated normally. Such terms arise naturally when one calculates the internal 
bending moment within a beam structure produced by uniformly distributed loading (UDL) when one has $n=2$, concentrated (or point) force loads when $n=1$, and point moments when $n=0$. Moreover, the load is located (or commences in the case of a UDL) at $x=a$, leading to an obvious mathematical structure. Having so derived an expression for the bending moment which, using this notation, is valid at any location along the beam, the moment-curvature relationship for the (small-slope) Euler-Bernoulli model is:

$$
M= \pm E I \frac{\mathrm{d}^{2} v}{\mathrm{~d} x^{2}}
$$

where the positive or negative sign depends upon the sign convention employed. This allows calculation of the transverse deflection, $v(x)$, by integrating relatively simple functions twice with respect to the axial coordinate, $x$. In practice, the integration is performed with respect to the argument of the bracket, rather than $x$, in order to keep the bracket and its meaning intact. For example, $x$ integrates as $x^{2} / 2$ in the normal way, but $[x-a]$ integrates as $[x-a]^{2} / 2$. Treated normally, $\int(x-a) \mathrm{d} x=x^{2} / 2-a x+C_{1}$, where $C_{1}$ is a constant, whereas if integrated with respect to the argument, $\int[x-a] \mathrm{d} x=[x-a]^{2} / 2+C_{2}$, where $C_{2}$ is also a constant. The difference lies in the value of the two constants of integration, the latter expression having the additional constant term $a^{2} / 2$; this difference is resolved so long as the constants are evaluated with the meaning of the brackets taken into account.

Macaulay's method is ideal for the calculation of the deflection of beam structures subject to a variety of loads along their span, including statically indeterminate systems. While the method is widely attributed to Macaulay [2], his being the first English-language description, Weissenburger [3] has provided some historical perspectives that indicate that the approach goes back to Clebsch in 1862. It has been generalised by Wittrick [4], who considered Euler-Bernoulli beams including axial compression and elastic foundations, as well as circular plates with a variety of discontinuous loads. Recent papers by Yavari et al. [5-8] have provided a variety of research results, including application to Timoshenko beams, elastic foundations and to cases in which the bending and shear stiffness properties change abruptly.

In this paper, Macaulay's method is applied to a Timoshenko beam, which includes the additional deflection due to shear. The level of treatment is appropriate to the practising engineer, or senior undergraduate, and it is assumed that the reader is familiar with the standard approach for the Euler-Bernoulli beam. The necessary modifications for the Timoshenko beam are illustrated by way of two examples, both of which are statically indeterminate. The first includes a variety of point force, moment and distributed loads, and is developed to the extent that the three equations necessary to calculate the reactions are generated; however, with such an array of loads, these equations are of considerable complexity and nothing is gained by proceeding to full expressions for the reactions. The second example concentrates on multiple point loads, which leads to more succinct expressions, and is developed to the stage of calculation of all reactions. Little new theory is required and it 
is sufficient to note that the governing equations for the Timoshenko beam model are:

$$
M=E I \frac{\mathrm{d} \psi}{\mathrm{d} x}
$$

and

$$
Q=\kappa A G\left(\frac{\mathrm{d} v}{\mathrm{~d} x}+\psi\right)
$$

where we employ the sign conventions from Reismann and Pawlik [9]. This implies that one requires expressions for both the bending moment and the shearing force that are valid at all locations along the beam.

Finally, note that Wang [10] has presented relationships between the slope, deflection and support reactions of a single-span Timoshenko beam, for a variety of end conditions, in terms of their Euler-Bernoulli counterparts. The present work shows that the Timoshenko beam can be treated from first principles in much the same way as a Euler-Bernoulli beam, with little added complexity.

\section{Example 1}

The first example is shown in Fig. 1 and consists of a propped cantilever beam subject to two point loads, a point moment and partial UDL. In Fig. 2, the complete structure is shown with the support reactions. Vertical force equilibrium requires:

$$
R_{A}+R_{B}=P_{1}+P_{2}+\frac{w L}{4}
$$

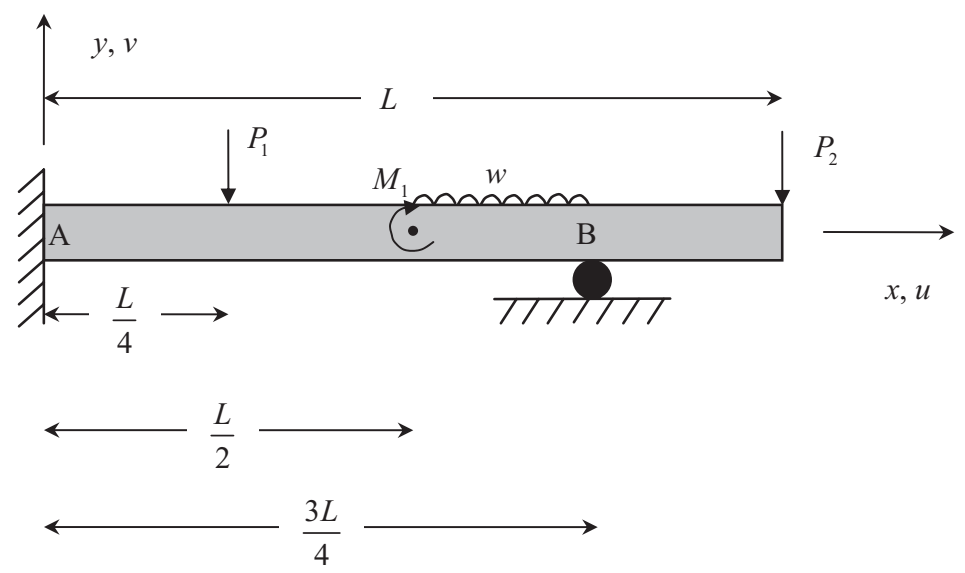

Fig. 1 Propped cantilever beam subject to point loads and moment, and a uniformly distributed load. 
Moment equilibrium (about the left-hand end) requires:

$$
M_{A}+R_{B} \frac{3 L}{4}=M_{1}+P_{1} \frac{L}{4}+P_{2} L+w\left(\frac{L}{4}\right)\left(\frac{5 L}{8}\right)
$$

At this stage one has three unknown reactions, but just two equations; the third is derived from the expression for the deflection curve.

Cut the beam at some generic cross-section $x$ close to the right-hand end, and insert a shearing force, $Q$, and a bending moment, $M$, as shown in Fig. 3. As is conventional, the UDL is continued on the upper surface, but negated by the introduction of an equal but opposite UDL on the lower surface. This has no effect on either the shearing force or the bending moment, but is necessary because the bracket notation 'switches on' the load, and an alternative device is required to switch it off. Vertical force equilibrium requires:

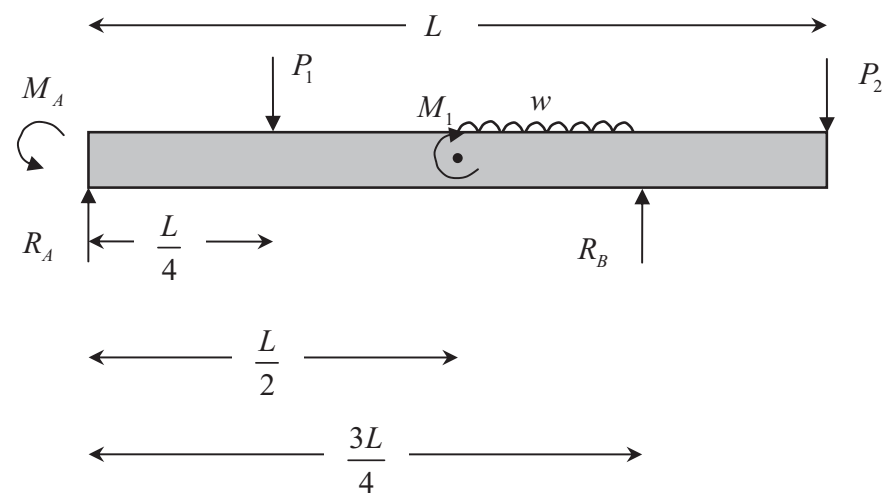

Fig. 2 Propped cantilever beam of Fig. 1, showing support reactions.

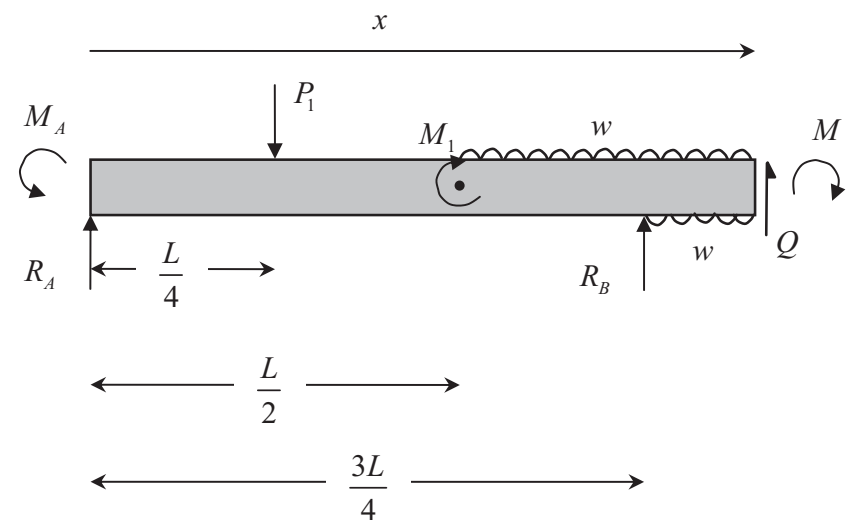

Fig. 3 Propped cantilever beam of Fig. 1 cut at generic cross-section $x$, with addition of shearing force, $Q$, and bending moment, $M$, on cut face. 


$$
Q+R_{A}+R_{B}\left[x-\frac{3 L}{4}\right]^{0}+w\left[x-\frac{3 L}{4}\right]=P_{1}\left[x-\frac{L}{4}\right]^{0}+w\left[x-\frac{L}{2}\right]
$$

Moment equilibrium about the cut face requires:

$$
\begin{aligned}
M & +M_{1}\left[x-\frac{L}{2}\right]^{0}+R_{A} x+R_{B}\left[x-\frac{3 L}{4}\right]+\frac{w}{2}\left[x-\frac{3 L}{4}\right]^{2} \\
& =P_{1}\left[x-\frac{L}{4}\right]+\frac{w}{2}\left[x-\frac{L}{2}\right]^{2}+M_{A}
\end{aligned}
$$

Now, set

$$
M=E I \frac{\mathrm{d} \psi}{\mathrm{d} x}
$$

and integrate to give:

$$
\begin{aligned}
& E I \psi+M_{1}\left[x-\frac{L}{2}\right]+\frac{R_{A} x^{2}}{2}+\frac{R_{B}}{2}\left[x-\frac{3 L}{4}\right]^{2}+\frac{w}{6}\left[x-\frac{3 L}{4}\right]^{2}= \\
& \frac{P_{1}}{2}\left[x-\frac{L}{4}\right]^{2}+\frac{w}{6}\left[x-\frac{L}{2}\right]^{3}+M_{A} x+C_{1}
\end{aligned}
$$

Apply the boundary condition $\psi(0)=0$ to give $C_{1}=0$.

Now rearrange the expression

$$
Q=\kappa A G\left(\psi+\frac{\mathrm{d} v}{\mathrm{~d} x}\right) \text { as } \frac{\mathrm{d} v}{\mathrm{~d} x}=\frac{Q}{\kappa A G}-\psi
$$

and substitute from equations 3 and 5 to give:

$$
\begin{aligned}
\frac{\mathrm{d} v}{\mathrm{~d} x}= & \frac{1}{\kappa A G}\left\{P_{1}\left[x-\frac{L}{4}\right]^{0}+w\left[x-\frac{L}{2}\right]-R_{A}-R_{B}\left[x-\frac{3 L}{4}\right]^{0}-w\left[x-\frac{3 L}{4}\right]\right\} \\
& -\frac{1}{E I}\left\{\frac{P_{1}}{2}\left[x-\frac{L}{4}\right]^{2}+\frac{w}{6}\left[x-\frac{L}{2}\right]^{3}+M_{A} x-M_{1}\left[x-\frac{L}{2}\right]-\frac{R_{A} x^{2}}{2}\right. \\
& \left.-\frac{R_{B}}{2}\left[x-\frac{3 L}{4}\right]^{2}-\frac{w}{6}\left[x-\frac{3 L}{4}\right]^{3}\right\}
\end{aligned}
$$

Integrate to give:

$$
\begin{aligned}
v(x)= & \frac{1}{\kappa A G}\left\{P_{1}\left[x-\frac{L}{4}\right]+\frac{w}{2}\left[x-\frac{L}{2}\right]^{2}-R_{A} x-R_{B}\left[x-\frac{3 L}{4}\right]-\frac{w}{2}\left[x-\frac{3 L}{4}\right]^{2}\right\} \\
& -\frac{1}{E I}\left\{\frac{P_{1}}{6}\left[x-\frac{L}{4}\right]^{3}+\frac{w}{24}\left[x-\frac{L}{2}\right]^{4}+\frac{M_{A} x^{2}}{2}-\frac{M_{1}}{2}\left[x-\frac{L}{2}\right]^{2}-\frac{R_{A} x^{3}}{6}\right. \\
& \left.-\frac{R_{B}}{6}\left[x-\frac{3 L}{4}\right]^{3}-\frac{w}{24}\left[x-\frac{3 L}{4}\right]^{4}\right\}+C_{2}
\end{aligned}
$$


Apply the boundary condition $v(0)=0$ to give $C_{2}=0$. The third equation necessary to determine the reactions derives from the further boundary condition $v\left(\frac{3 L}{4}\right)=0$,
to give:

$$
\begin{aligned}
0= & \frac{1}{\kappa A G}\left\{P_{1}\left(\frac{L}{2}\right)+\frac{w}{2}\left(\frac{L}{4}\right)^{2}-R_{A}\left(\frac{3 L}{4}\right)\right\}-\frac{1}{E I}\left\{\frac{P_{1}}{6}\left(\frac{L}{2}\right)^{3}+\frac{w}{24}\left(\frac{L}{4}\right)^{4}+\frac{M_{A}}{2}\left(\frac{3 L}{4}\right)^{2}\right. \\
& \left.-\frac{M_{1}}{2}\left(\frac{L}{4}\right)^{2}-\frac{R_{A}}{6}\left(\frac{3 L}{4}\right)^{3}\right\}
\end{aligned}
$$

While equation 8 can be simplified by the introduction of a dimensionless stiffness parameter of the form $\left(E I / \kappa A G L^{2}\right)$, these equations are of considerable complexity and little is gained by proceeding to full expressions for the reactions, and the analysis is not pursued further.

\section{Example 2}

The second example is also a propped cantilever beam, now subject to multiple point loading, as shown, with support reactions, in Fig. 4. Vertical force equilibrium requires:

$$
R_{0}+R_{7}=10 P
$$

Moment equilibrium (about the left-hand end) requires:

$$
M_{0}+7 R_{7}=(1+2+\ldots+10) P=55 P
$$

Again, one has three unknown reactions, but just two equations, with the third derived from the expression for the deflection curve.

Again, the beam is cut at some generic cross-section $x$ close to the right-hand end, and a shearing force, $Q$, and a bending moment, $M$, are inserted, as shown in Fig. 5. Vertical equilibrium requires:

$$
Q+R_{0}+R_{7}[x-7]^{0}=P\left\{[x-1]^{0}+[x-2]^{0}+\ldots+[x-9]^{0}\right\}
$$

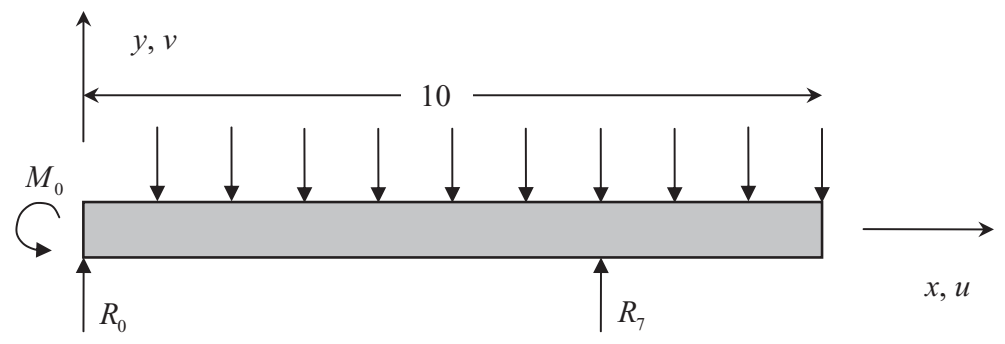

Fig. 4 Propped cantilever beam with 10 equi-spaced point loads, and simple support at $x=7$, showing support reactions. Each downward arrow represents a force of magnitude $P$. 


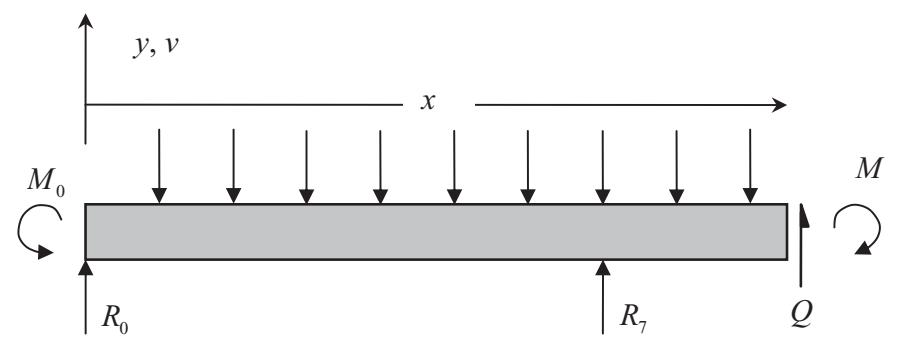

Fig. 5 Propped cantilever beam of Fig. 4 cut at generic cross-section $x$, with addition of shearing force, $Q$, and bending moment, $M$, on cut face.

Moment equilibrium requires

$$
M+R_{0} x+R_{7}[x-7]=M_{0}+P\{[x-1]+[x-2]+\ldots+[x-9]\}
$$

Again, set

$$
M=E I \frac{\mathrm{d} \psi}{\mathrm{d} x}
$$

in equation 12 and integrate to give:

$$
E I \psi+\frac{R_{0} x^{2}}{2}+\frac{R_{7}}{2}[x-7]^{2}=M_{0} x+\frac{P}{2}\left\{[x-1]^{2}+[x-2]^{2}+\ldots+[x-9]^{2}\right\}+C_{1}
$$

Again, the constant $C_{1}=0$, on account of the boundary condition at the left-hand end, $\psi(0)=0$. Now, construct

$$
\frac{\mathrm{d} v}{\mathrm{~d} x}=\frac{Q}{\kappa A G}-\psi
$$

and integrate to give:

$$
\begin{aligned}
v(x) & =\frac{P}{\kappa A G}\{[x-1]+[x-2]+\ldots+[x-9]\}-\frac{R_{0} x}{\kappa A G}-\frac{R_{7}}{\kappa A G}[x-7] \\
& -\frac{M_{0} x^{2}}{2 E I}+\frac{R_{0} x^{3}}{6 E I}+\frac{R_{7}}{6 E I}[x-7]^{3}-\frac{P}{6 E I}\left\{[x-1]^{3}+[x-2]^{3}+\ldots+[x-9]^{3}\right\}+C_{2}
\end{aligned}
$$

And again, the constant $C_{2}=0$, on account of the boundary condition at the left-hand end, $v(0)=0$.

The necessary third equation derives from the further boundary condition, $v(7)=0$, to give:

$$
0=\frac{P}{\kappa A G}\{6+5+\ldots+1\}-\frac{7 R_{0}}{\kappa A G}-\frac{7^{2} M_{0}}{2 E I}+\frac{7^{3} R_{0}}{6 E I}-\frac{P}{6 E I}\left\{6^{3}+5^{3}+\ldots+1^{3}\right\}
$$

The reactions are then calculated as: 


$$
R_{7}=\frac{7 P}{(1+\gamma)}\left(\frac{52}{7^{2}}+\gamma\right), \quad R_{0}=\frac{3 P}{1+\gamma}\left(\frac{6}{7}+\gamma\right), \quad M_{0}=\frac{3 P}{1+\gamma}(1+2 \gamma)
$$

where $\gamma=\frac{3 E I}{7^{2} \kappa A G}$

Note that the reactions for the Euler-Bernoulli beam are found by setting $\gamma=0$. It is now straightforward to substitute into equations 11,12 or 14 to give expressions for the shearing force, bending moment and transverse deflection that are valid at all locations along the beam.

\section{Conclusions}

By way of two worked examples, Macaulay's method has been extended to the deflection analysis of the Timoshenko beam model. One now requires general expressions for both the bending moment, as in the treatment of a Euler-Bernoulli beam, and the shearing force that are valid at all locations along the beam.

\section{References}

[1] P. P. Benham, R. J. Crawford and C. G. Armstrong, Mechanics of Engineering Materials (2nd edition) (Longman, Harlow, 1996).

[2] W. H. Macaulay, 'A note on the deflection of beams', Messenger of Mathematics, 48 (1919), 129.

[3] J. T. Weissenburger, 'Integration of discontinuous expressions arising in beam theory', AIAA Journal, 2(1) (1964), 106-108.

[4] W. H. Wittrick, 'A generalization of Macaulay's method with applications in structural mechanics', AIAA Journal, 3(2) (1965), 326-330.

[5] A. Yavari, S. Sarkani and E. T. Moyer Jr, 'On applications of generalized functions to beam bending problems', International Journal of Solids and Structures, 37(40) (2000), 5675-5705.

[6] A. Yavari, S. Sarkani and J. N. Reddy, 'Generalised solutions of beams with jump discontinuities on elastic foundations', Archive of Applied Mechanics, 71(9) (2001), 625-639.

[7] A. Yavari, S. Sarkani and J. N. Reddy, 'On nonuniform Euler-Bernoulli and Timoshenko beams with jump discontinuities: application of distribution theory', International Journal of Solids and Structures, 38(46-7) (2001), 8389-8406.

[8] A. Yavari and S. Sarkani, 'On applications of generalized functions to the analysis of EulerBernoulli beam-columns with jump discontinuities', International Journal of Mechanical Sciences, 43(6) (2001), 1543-1562.

[9] H. Reismann and P. S. Pawlik, Elasticity, Theory and Applications (Wiley, Chichester, 1980), pp. 221-222.

[10] C. M. Wang, 'Timoshenko beam-bending solutions in terms of Euler-Bernoulli solutions', Journal of Engineering Mechanics, 121(6) (1995), 763-765. 